quatrième série - tome $43 \quad$ fascicule 2 mars-avril 2010

$$
\begin{aligned}
& \text { ANNALES } \\
& \text { SCIENTIFIQUES } \\
& \text { de } \\
& \text { L'ECOLE } \\
& \text { NORMALE } \\
& \text { SUPÉRIEURE }
\end{aligned}
$$

Kazuma MORITA

Hodge-Tate and de Rham representations

in the imperfect residue field case 
Ann. Scient. Éc. Norm. Sup.

$4^{\mathrm{e}}$ série, t. 43,2010 , p. 341 à 355

\title{
HODGE-TATE AND DE RHAM REPRESENTATIONS IN THE IMPERFECT RESIDUE FIELD CASE
}

\author{
BY KaZuma MORITA
}

\begin{abstract}
Let $K$ be a $p$-adic local field with residue field $k$ such that $\left[k: k^{p}\right]=p^{e}<+\infty$ and $V$ be a $p$-adic representation of $\operatorname{Gal}(\bar{K} / K)$. Then, by using the theory of $p$-adic differential modules, we show that $V$ is a Hodge-Tate (resp. de Rham) representation of $\mathrm{Gal}(\bar{K} / K)$ if and only if $V$ is a Hodge-Tate (resp. de Rham) representation of $\operatorname{Gal}\left(\overline{K^{\mathrm{pf}}} / K^{\mathrm{pf}}\right)$ where $K^{\mathrm{pf}} / K$ is a certain $p$-adic local field with residue field the smallest perfect field $k^{\mathrm{pf}}$ containing $k$.

RÉsumé. - Soit $K$ un corps local $p$-adique de corps résiduel $k$ tel que $\left[k: k^{p}\right]=p^{e}<+\infty$ et soit $V$ une représentation $p$-adique de $\operatorname{Gal}(\bar{K} / K)$. Nous utilisons la théorie des modules différentiels $p$-adiques pour montrer que $V$ est une représentation de Hodge-Tate (resp. de Rham) de $\mathrm{Gal}(\bar{K} / K)$ si et seulement si $V$ est une représentation de Hodge-Tate (resp. de Rham) de $\operatorname{Gal}\left(\overline{K^{\mathrm{pf}}} / K^{\mathrm{pf}}\right)$ où $K^{\mathrm{pf}} / K$ est un certain corps local $p$-adique de corps résiduel le plus petit corps parfait $k^{\mathrm{pf}}$ contenant $k$.
\end{abstract}

\section{Introduction}

Let $K$ be a complete discrete valuation field of characteristic 0 with residue field $k$ of characteristic $p>0$ such that $\left[k: k^{p}\right]=p^{e}<+\infty$. Choose an algebraic closure $\bar{K}$ of $K$ and put $G_{K}=\operatorname{Gal}(\bar{K} / K)$. By a $p$-adic representation of $G_{K}$, we mean a finite dimensional vector space $V$ over $\mathbb{Q}_{p}$ endowed with a continuous action of $G_{K}$. In the case $e=0$ (i.e. $k$ is perfect), following Fontaine, we can classify $p$-adic representations of $G_{K}$ by using the $p$-adic periods rings $B_{\mathrm{HT}}, B_{\mathrm{dR}}, B_{\mathrm{st}}$ and $B_{\text {cris }}$ (Hodge-Tate, de Rham, semi-stable and crystalline representations). In the general case (i.e. $k$ is not necessarily perfect), Hyodo constructed the imperfect residue field version of the ring $B_{\mathrm{HT}}$ and Tsuzuki and several authors constructed that of the ring $B_{\mathrm{dR}}$. By using these rings, we can define the imperfect residue field version of Hodge-Tate and de Rham representations of $G_{K}$ in the evident way ([3], [7], [8], [9], [12]).

Now, we shall state the main result of this article. Let us fix some notations. Fix a lifting $\left(b_{i}\right)_{1 \leq i \leq e}$ of a $p$-basis of $k$ in $\mathscr{O}_{K}$ (the ring of integers of $K$ ) and for each $m \geq 1$, fix a $p^{m}$-th $\operatorname{root} b_{i}^{1 / p^{m}}$ of $b_{i}$ in $\bar{K}$ satisfying $\left(b_{i}^{1 / p^{m}+1}\right)^{p}=b_{i}^{1 / p^{m}}$. Put $K^{(\mathrm{pf})}=\cup_{m \geq 1} K\left(b_{i}^{1 / p^{m}}, 1 \leq i \leq e\right)$ and $K^{\mathrm{pf}}=$ the $p$-adic completion of $K^{(\mathrm{pf})}$. These fields depend on the choice of a lifting 
of a $p$-basis of $k$ in $\mathscr{O}_{K}$. Since $K^{\mathrm{pf}}$ becomes a complete discrete valuation field with perfect residue field, we can apply theories in the perfect residue field case to $p$-adic representations of $G_{K^{\mathrm{pf}}}=\operatorname{Gal}\left(\overline{K^{\mathrm{pf}}} / K^{\mathrm{pf}}\right)$ where we choose an algebraic closure $\overline{K^{\mathrm{pf}}}$ of $K^{\mathrm{pf}}$ containing $\bar{K}$. Note that, if $V$ is a $p$-adic representation of $G_{K}$, it can be also regarded as a $p$-adic representation of $G_{K^{\mathrm{pf}}}$ (see Section 2.2 for details). Our main result is the following.

Theorem 1.1. - Let $K$ be a complete discrete valuation field of characteristic 0 with residue field $k$ of characteristic $p>0$ such that $\left[k: k^{p}\right]=p^{e}<+\infty$ and $V$ be a $p$-adic representation of $G_{K}$. Let $K^{\mathrm{pf}}$ be the field extension of $K$ defined as above. Then, we have the following equivalences

1. $V$ is a Hodge-Tate representation of $G_{K}$ if and only if $V$ is a Hodge-Tate representation of $G_{K^{\mathrm{p}}}$,

2. $V$ is a de Rham representation of $G_{K}$ if and only if $V$ is a de Rham representation of $G_{K^{\mathrm{p}}}$.

In the case of Hodge-Tate representations, Tsuji [11] had proved a more refined theorem based on this article. This paper is organized as follows. In Section 2, we shall review the definitions and basic known facts on Hodge-Tate and de Rham representations, first in the perfect residue field case and then in the imperfect residue field case. In Section 3, we shall review the theory of $p$-adic differential modules which play a central role in this article. In Section 4 , by using the theory of $p$-adic differential modules, we shall prove the main theorem, first for Hodge-Tate representations and then for de Rham representations.

\section{Acknowledgments}

The author would like to thank his advisor Professor Kazuya Kato for continuous advice, encouragements and patience. He also would like to thank Marc-Hubert Nicole and Shun Okubo for reading a manuscript carefully and giving useful comments. A part of this work was done while he was staying at Universite Paris-Sud 11 and he thanks this institute for its hospitality. His staying at Université Paris-Sud 11 is partially supported by JSPS Core-toCore Program "New Developments of Arithmetic Geometry, Motive, Galois Theory, and their practical applications" and he thanks Professor Makoto Matsumoto for encouraging this visiting. This research is partially supported by Grand-in-Aid for Young Scientists Start-up.

\section{Preliminaries on Hodge-Tate and de Rham representations}

\subsection{Hodge-Tate and de Rham representations in the perfect residue field case}

(See [4] and [5] for details.) Let $K$ be a complete discrete valuation field of characteristic 0 with perfect residue field $k$ of characteristic $p>0$. Choose an algebraic closure $\bar{K}$ of $K$ and consider its $p$-adic completion $\mathbb{C}_{p}$. Put

$$
\widetilde{\mathbb{E}}=\lim _{x \mapsto x^{p}} \mathbb{C}_{p}=\left\{\left(x^{(0)}, x^{(1)}, \ldots\right) \mid\left(x^{(i+1)}\right)^{p}=x^{(i)}, x^{(i)} \in \mathbb{C}_{p}\right\}
$$

and let $\widetilde{\mathbb{E}}^{+}$denote the set of $x=\left(x^{(i)}\right) \in \widetilde{\mathbb{E}}$ such that $x^{(0)} \in \mathscr{O}_{\mathbb{C}_{p}}$ where $\mathscr{O}_{\mathbb{C}_{p}}$ denotes the ring of integers of $\mathbb{C}_{p}$. For two elements $x=\left(x^{(i)}\right)$ and $y=\left(y^{(i)}\right)$ of $\widetilde{\mathbb{E}}$, their sum and 
product are defined by $(x+y)^{(i)}=\lim _{j \rightarrow+\infty}\left(x^{(i+j)}+y^{(i+j)}\right)^{p^{j}}$ and $(x y)^{(i)}=x^{(i)} y^{(i)}$. These sum and product make $\widetilde{\mathbb{E}}$ a perfect field of characteristic $p>0\left(\widetilde{\mathbb{E}}^{+}\right.$is a subring of $\left.\widetilde{\mathbb{E}}\right)$. Let $\epsilon=\left(\epsilon^{(n)}\right)$ be an element of $\widetilde{\mathbb{E}}$ such that $\epsilon^{(0)}=1$ and $\epsilon^{(1)} \neq 1$. Then, $\widetilde{\mathbb{E}}$ is the completion of an algebraic closure of $k((\epsilon-1))$ for the valuation defined by $v_{\mathbb{E}}(x)=v_{p}\left(x^{(0)}\right)$ where $v_{p}$ denotes the $p$-adic valuation of $\mathbb{C}_{p}$ normalized by $v_{p}(p)=1$. The field $\widetilde{\mathbb{E}}$ is equipped with a continuous action of the Galois group $G_{K}=\mathrm{Gal}(\bar{K} / K)$ with respect to the topology defined by the valuation $v_{\mathbb{E}}$. Put $\widetilde{\mathbb{A}}^{+}=W\left(\widetilde{\mathbb{E}}^{+}\right)$(the ring of Witt vectors with coefficients in $\left.\widetilde{\mathbb{E}}^{+}\right)$and $\widetilde{\mathbb{B}}^{+}=\widetilde{\mathbb{A}}^{+}[1 / p]=\left\{\sum_{k \gg-\infty} p^{k}\left[x_{k}\right] \mid x_{k} \in \widetilde{\mathbb{E}}^{+}\right\}$where $[*]$ denotes the Teichmüller lift of $* \in \widetilde{\mathbb{E}}^{+}$. This ring $\widetilde{\mathbb{B}}^{+}$is equipped with a surjective homomorphism

$$
\theta: \widetilde{\mathbb{B}}^{+} \rightarrow \mathbb{C}_{p}: \sum p^{k}\left[x_{k}\right] \mapsto \sum p^{k} x_{k}^{(0)} .
$$

If $\tilde{p}=\left(p^{(n)}\right)$ denotes an element of $\widetilde{\mathbb{E}}^{+}$such that $p^{(0)}=p$, we can show that $\operatorname{Ker}(\theta)$ is the principal ideal generated by $\omega=[\tilde{p}]-p$. The $\operatorname{ring} B_{\mathrm{dR}, K}^{+}$is defined to be the $\operatorname{Ker}(\theta)$-adic completion of $\widetilde{\mathbb{B}}^{+}$

$$
B_{\mathrm{dR}, K}^{+}=\varliminf_{n \geq 0} \widetilde{\mathbb{B}}^{+} /\left(\operatorname{Ker}(\theta)^{n}\right) .
$$

This is a discrete valuation ring and $t=\log ([\epsilon])$ which converges in $B_{\mathrm{dR}, K}^{+}$is a generator of the maximal ideal. Put $B_{\mathrm{dR}, K}=B_{\mathrm{dR}, K}^{+}[1 / t]$. This ring $B_{\mathrm{dR}, K}$ becomes a field and is equipped with an action of the Galois group $G_{K}$ and a filtration defined by $\mathrm{Fil}^{i} B_{\mathrm{dR}, K}=t^{i} B_{\mathrm{dR}, K}^{+}(i \in \mathbb{Z})$. Then, $\left(B_{\mathrm{dR}, K}\right)^{G_{K}}$ is canonically isomorphic to $K$. Thus, for a $p$-adic representation $V$ of $G_{K}, D_{\mathrm{dR}, K}(V)=\left(B_{\mathrm{dR}, K} \otimes_{\mathbb{Q}_{p}} V\right)^{G_{K}}$ is naturally a $K$-vector space. We say that a $p$-adic representation $V$ of $G_{K}$ is a de Rham representation of $G_{K}$ if we have

$$
\operatorname{dim}_{\mathbb{Q}_{p}} V=\operatorname{dim}_{K} D_{\mathrm{dR}, K}(V) \quad\left(\text { we always have } \operatorname{dim}_{\mathbb{Q}_{p}} V \geq \operatorname{dim}_{K} D_{\mathrm{dR}, K}(V)\right) .
$$

Furthermore, we say that a $p$-adic representation $V$ of $G_{K}$ is a potentially de Rham representation of $G_{K}$ if there exists a finite field extension $L / K$ in $\bar{K}$ such that $V$ is a de Rham representation of $G_{L}$. It is known that a potentially de Rham representation $V$ of $G_{K}$ is a de Rham representation of $G_{K}$ (see [5, 3.9]).

Define $B_{\mathrm{HT}, K}$ to be the associated graded algebra to the filtration $\mathrm{Fil}^{i} B_{\mathrm{dR}, K}$. The quotient $\mathrm{gr}^{i} B_{\mathrm{HT}, K}=\mathrm{Fil}^{i} B_{\mathrm{dR}, K} / \mathrm{Fil}^{i+1} B_{\mathrm{dR}, K}(i \in \mathbb{Z})$ is a one-dimensional $\mathbb{C}_{p}$-vector space spanned by the image of $t^{i}$. Thus, we obtain the presentation

$$
B_{\mathrm{HT}, K}=\bigoplus_{i \in \mathbb{Z}} \mathbb{C}_{p}(i)
$$

where $\mathbb{C}_{p}(i)=\mathbb{C}_{p} \otimes \mathbb{Z}_{p}(i)$ is the Tate twist. Then, $\left(B_{\mathrm{HT}, K}\right)^{G_{K}}$ is canonically isomorphic to $K$. Thus, for a $p$-adic representation $V$ of $G_{K}, D_{\mathrm{HT}, K}(V)=\left(B_{\mathrm{HT}, K} \otimes_{\mathbb{Q}_{p}} V\right)^{G_{K}}$ is naturally a $K$-vector space. We say that a $p$-adic representation $V$ of $G_{K}$ is a Hodge-Tate representation of $G_{K}$ if we have

$$
\operatorname{dim}_{\mathbb{Q}_{p}} V=\operatorname{dim}_{K} D_{\mathrm{HT}, K}(V) \quad\left(\text { we always have } \operatorname{dim}_{\mathbb{Q}_{p}} V \geq \operatorname{dim}_{K} D_{\mathrm{HT}, K}(V)\right) .
$$

Furthermore, we say that a $p$-adic representation $V$ of $G_{K}$ is a potentially Hodge-Tate representation of $G_{K}$ if there exists a finite field extension $L / K$ in $\bar{K}$ such that $V$ is a Hodge-Tate representation of $G_{L}$. It is known that a potentially Hodge-Tate representation $V$ of $G_{K}$ is a Hodge-Tate representation of $G_{K}$ (see [5, 3.9]). Since we have $\operatorname{gr} B_{\mathrm{dR}, K} \simeq \bigoplus_{i \in \mathbb{Z}} \mathbb{C}_{p}(i)$, 
if $V$ is a de Rham representation of $G_{K}$, there exists a $G_{K}$-equivariant isomorphism $\mathbb{C}_{p} \otimes_{\mathbb{Q}_{p}} V \simeq \bigoplus_{j=1}^{d=\operatorname{dim}_{\mathbb{Q}_{p}} V} \mathbb{C}_{p}\left(n_{j}\right)\left(n_{j} \in \mathbb{Z}\right)$. Thus, it follows that a de Rham representation $V$ of $G_{K}$ is a Hodge-Tate representation of $G_{K}$.

\subsection{Hodge-Tate and de Rham representations in the imperfect residue field case}

Let $K$ be a complete discrete valuation field of characteristic 0 with residue field $k$ of characteristic $p>0$ such that $\left[k: k^{p}\right]=p^{e}<+\infty$. Choose an algebraic closure $\bar{K}$ of $K$ and put $G_{K}=\operatorname{Gal}(\bar{K} / K)$. As in the introduction, fix a lifting $\left(b_{i}\right)_{1 \leq i \leq e}$ of a $p$-basis of $k$ in $\mathscr{O}_{K}$ (the ring of integers of $K$ ) and for each $m \geq 1$, fix a $p^{m}$-th root $b_{i}^{1 / p^{m}}$ of $b_{i}$ in $\bar{K}$ satisfying $\left(b_{i}^{1 / p^{m+1}}\right)^{p}=b_{i}^{1 / p^{m}}$. Put

$$
K^{(\mathrm{pf})}=\cup_{m \geq 0} K\left(b_{i}^{1 / p^{m}}, 1 \leq i \leq e\right) \quad \text { and } \quad K^{\mathrm{pf}}=\text { the } p \text {-adic completion of } K^{(\mathrm{pf})} .
$$

These fields depend on the choice of a lifting of a $p$-basis of $k$ in $\mathscr{O}_{K}$. Since $K^{(\mathrm{pf})}$ is a Henselian discrete valuation field, we have an isomorphism $G_{K^{\mathrm{pf}}}=\operatorname{Gal}\left(\overline{K^{\mathrm{pf}}} / K^{\mathrm{pf}}\right) \simeq$ $G_{K^{(\mathrm{pf})}}=\operatorname{Gal}\left(\bar{K} / K^{(\mathrm{pf})}\right)\left(\subset G_{K}\right)$ where we choose an algebraic closure $\overline{K^{\mathrm{pf}}}$ of $K^{\mathrm{pf}}$ containing $\bar{K}$. With this isomorphism, we identify $G_{K^{\mathrm{pf}}}$ with a subgroup of $G_{K}$. We have a bijective map from the set of finite extensions of $K^{(\mathrm{pf})}$ contained in $\bar{K}$ to the set of finite extensions of $K^{\mathrm{pf}}$ contained in $\overline{K^{\mathrm{pf}}}$ defined by $L \rightarrow L K^{\mathrm{pf}}$. Furthermore, $L K^{\mathrm{pf}}$ is the $p$-adic completion of $L$. Hence, we have an isomorphism of rings

$$
\mathscr{O}_{\bar{K}} / p^{n} \mathscr{O}_{\bar{K}} \simeq \mathscr{O}_{\overline{K^{\mathrm{pf}}}} / p^{n} \mathscr{O}_{\overline{K^{\mathrm{pf}}}}
$$

where $\mathscr{O}_{\bar{K}}$ and $\mathscr{O}_{\overline{K^{\mathrm{pf}}}}$ denote the rings of integers of $\bar{K}$ and $\overline{K^{\mathrm{pf}}}$. Thus, the $p$-adic completion of $\bar{K}$ is isomorphic to the $p$-adic completion of $\overline{K^{\mathrm{pf}}}$, which we will write $\mathbb{C}_{p}$. As in Subsection 2.1 , construct the rings $\widetilde{\mathbb{E}}^{+}$and $\widetilde{\mathbb{A}}+=W\left(\widetilde{\mathbb{E}}^{+}\right)$from this $\mathbb{C}_{p}$. Let $k^{\mathrm{pf}}$ denote the perfect residue field of $K^{\mathrm{pf}}$ and put $\mathscr{O}_{K_{0}}=\mathscr{O}_{K} \cap W\left(k^{\mathrm{pf}}\right)$. Let $\alpha: \mathscr{O}_{K} \otimes_{\mathscr{O}_{K_{0}}} \widetilde{\mathbb{A}}^{+} \rightarrow \mathscr{O}_{\bar{K}} / p \mathscr{O}_{\bar{K}}$ be the natural surjection and define $\widetilde{\mathbb{A}}_{(K)}^{+}$to be $\widetilde{\mathbb{A}}_{(K)}^{+}=\varliminf_{n \geq 0}\left(\mathscr{O}_{K} \otimes_{\mathscr{O}_{K_{0}}} \widetilde{\mathbb{A}}^{+}\right) /(\operatorname{Ker}(\alpha))^{n}$. Let $\theta_{K}: \widetilde{\mathbb{A}}_{(K)}^{+} \otimes_{\mathbb{Z}_{p}} \mathbb{Q}_{p} \rightarrow \mathbb{C}_{p}$ be the natural extension of $\theta: \widetilde{\mathbb{A}}^{+}[1 / p] \rightarrow \mathbb{C}_{p}$. Define $B_{\mathrm{dR}, K}^{+}$to be the $\operatorname{Ker}\left(\theta_{K}\right)$-adic completion of $\widetilde{\mathbb{A}}_{(K)}^{+} \otimes_{\mathbb{Z}_{p}} \mathbb{Q}_{p}$

$$
B_{\mathrm{dR}, K}^{+}=\lim _{n \geq 0}\left(\widetilde{\mathbb{A}}_{(K)}^{+} \otimes_{\mathbb{Z}_{p}} \mathbb{Q}_{p}\right) /\left(\operatorname{Ker}\left(\theta_{K}\right)^{n}\right) .
$$

This is a $K$-algebra equipped with an action of the Galois group $G_{K}$. Let $\widetilde{b}_{i}$ denote $\left(b_{i}^{(n)}\right) \in \widetilde{\mathbb{E}}^{+}$such that $b_{i}^{(0)}=b_{i}$ and then the series which defines $\log \left(\left[\widetilde{b}_{i}\right] / b_{i}\right)$ converges to an element $t_{i}$ in $B_{\mathrm{dR}, K}^{+}$. Then, the ring $B_{\mathrm{dR}, K}^{+}$becomes a local ring with the maximal ideal $m_{\mathrm{dR}}=\left(t, t_{1}, \ldots, t_{e}\right)$. Define a filtration on $B_{\mathrm{dR}, K}^{+}$by fil ${ }^{i} B_{\mathrm{dR}, K}^{+}=m_{\mathrm{dR}}^{i}$. Then, the homomorphism

$$
f: B_{\mathrm{dR}, K^{\mathrm{pf}}}^{+}\left[\left[t_{1}, \ldots, t_{e}\right]\right] \rightarrow B_{\mathrm{dR}, K}^{+}
$$

is an isomorphism of filtered algebras (see [3, Proposition 2.9]). From this isomorphism, it follows easily that

$$
i: B_{\mathrm{dR}, K^{\mathrm{pf}}}^{+} \hookrightarrow B_{\mathrm{dR}, K}^{+} \quad \text { and } \quad p: B_{\mathrm{dR}, K}^{+} \rightarrow B_{\mathrm{dR}, K^{\mathrm{pf}}}^{+}: t_{i} \mapsto 0
$$

are $G_{K^{\mathrm{pf}}}$-equivariant homomorphisms and the composition

$$
p \circ i: B_{\mathrm{dR}, K^{\mathrm{pf}}}^{+} \hookrightarrow B_{\mathrm{dR}, K}^{+} \rightarrow B_{\mathrm{dR}, K^{\mathrm{pf}}}^{+}
$$

$4^{\mathrm{e}}$ SÉRIE - TOME $43-2010-\mathrm{N}^{\mathrm{o}} 2$ 
is an identity. Put $B_{\mathrm{dR}, K}=B_{\mathrm{dR}, K}^{+}[1 / t]$. Then, $K$ is canonically embedded in $B_{\mathrm{dR}, K}$ and we have a canonical isomorphism $\left(B_{\mathrm{dR}, K}\right)^{G_{K}}=K$. Thus, for a $p$-adic representation $V$ of $G_{K}, D_{\mathrm{dR}, K}(V)=\left(B_{\mathrm{dR}, K} \otimes_{\mathbb{Q}_{p}} V\right)^{G_{K}}$ is naturally a $K$-vector space. We say that a $p$-adic representation $V$ of $G_{K}$ is a de Rham representation of $G_{K}$ if we have

$$
\operatorname{dim}_{\mathbb{Q}_{p}} V=\operatorname{dim}_{K} D_{\mathrm{dR}, K}(V) \quad \text { (we always have } \operatorname{dim}_{\mathbb{Q}_{p}} V \geq \operatorname{dim}_{K} D_{\mathrm{dR}, K}(V) \text { ). }
$$

Furthermore, we say that a $p$-adic representation $V$ of $G_{K}$ is a potentially de Rham representation of $G_{K}$ if there exists a finite field extension $L / K$ in $\bar{K}$ such that $V$ is a de Rham representation of $G_{L}$. We can show that a potentially de Rham representation $V$ of $G_{K}$ is a de Rham representation of $G_{K}$ in the same way as in the perfect residue field case.

Define a filtration on $B_{\mathrm{dR}, K}$ to be

$$
\begin{aligned}
\mathrm{Fil}^{0} B_{\mathrm{dR}, K} & =\sum_{n=0}^{\infty} t^{-n} \mathrm{fil}^{n} B_{\mathrm{dR}, K}^{+}=B_{\mathrm{dR}, K}^{+}\left[\frac{t_{1}}{t}, \ldots, \frac{t_{e}}{t}\right], \\
\mathrm{Fil}^{i} B_{\mathrm{dR}, K} & =t^{i} \mathrm{Fil}^{0} B_{\mathrm{dR}, K}(i \in \mathbb{Z}) .
\end{aligned}
$$

Define $B_{\mathrm{HT}, K}$ to be the associated graded algebra to this filtration. Since the quotient $\operatorname{gr}^{i} B_{\mathrm{HT}, K}=\mathrm{Fil}^{i} B_{\mathrm{dR}, K} / \mathrm{Fil}^{i+1} B_{\mathrm{dR}, K}(i \in \mathbb{Z})$ is given by $\operatorname{gr}^{i} B_{\mathrm{HT}, K}=t^{i} \mathbb{C}_{p}\left[\frac{t_{1}}{t}, \ldots, \frac{t_{e}}{t}\right]$, we obtain the presentation

$$
B_{\mathrm{HT}, K}=\mathbb{C}_{p}\left[t, t^{-1}, \frac{t_{1}}{t}, \ldots, \frac{t_{e}}{t}\right]=B_{\mathrm{HT}, K^{\mathrm{pf}}}\left[\frac{t_{1}}{t}, \ldots, \frac{t_{e}}{t}\right] .
$$

From this presentation, it follows easily that

$$
i: B_{\mathrm{HT}, K^{\mathrm{pf}}} \hookrightarrow B_{\mathrm{HT}, K} \quad \text { and } \quad p: B_{\mathrm{HT}, K} \rightarrow B_{\mathrm{HT}, K^{\mathrm{pf}}}: t_{i} / t \mapsto 0
$$

are $G_{K^{\text {pf }}}$-equivariant homomorphisms and the composition

$$
p \circ i: B_{\mathrm{HT}, K^{\mathrm{pf}}} \hookrightarrow B_{\mathrm{HT}, K} \rightarrow B_{\mathrm{HT}, K^{\mathrm{pf}}}
$$

is an identity. The field $K$ is canonically embedded in $B_{\mathrm{HT}, K}$ and we have $\left(B_{\mathrm{HT}, K}\right)^{G_{K}}=K$. Thus, for a $p$-adic representation $V$ of $G_{K}, D_{\mathrm{HT}, K}(V)=\left(B_{\mathrm{HT}, K} \otimes_{\mathbb{Q}_{p}} V\right)^{G_{K}}$ is naturally a $K$-vector space. We say that a $p$-adic representation $V$ of $G_{K}$ is a Hodge-Tate representation of $G_{K}$ if we have

$$
\operatorname{dim}_{\mathbb{Q}_{p}} V=\operatorname{dim}_{K} D_{\mathrm{HT}, K}(V) \quad \text { (we always have } \operatorname{dim}_{\mathbb{Q}_{p}} V \geq \operatorname{dim}_{K} D_{\mathrm{HT}, K}(V) \text { ). }
$$

Furthermore, we say that a $p$-adic representation $V$ of $G_{K}$ is a potentially Hodge-Tate representation of $G_{K}$ if there exists a finite field extension $L / K$ in $\bar{K}$ such that $V$ is a HodgeTate representation of $G_{L}$. We can show that a potentially Hodge-Tate representation $V$ of $G_{K}$ is a Hodge-Tate representation of $G_{K}$ in the same way as in the perfect residue field case.

\section{Preliminaries on $p$-adic differential modules}

In this section, we shall review the theory of $p$-adic differential modules which plays an important role in this article. First, let us fix the notations. Let $K$ be a complete discrete valuation field of characteristic 0 with residue field $k$ of characteristic $p>0$ such that $\left[k: k^{p}\right]=p^{e}<\infty$ and $V$ be a $p$-adic representation of $G_{K}$. Define $K^{(\mathrm{pf})}$ and $K^{\mathrm{pf}}$ as in the introduction and in Subsection 2.2. Put $K_{\infty}^{(\mathrm{pf})}=\cup_{m \geq 0} K^{(\mathrm{pf})}\left(\zeta_{p^{m}}\right)$ (resp. $K_{\infty}^{\mathrm{pf}}=$ $\cup_{m \geq 0} K^{\mathrm{pf}}\left(\zeta_{p^{m}}\right)$ ) where $\zeta_{p^{m}}$ denotes a primitive $p^{m}$-th root of unity in $\bar{K}$ (resp. $\overline{K^{\mathrm{pf}}}$ ) such 
that $\left(\zeta_{p^{m+1}}\right)^{p}=\zeta_{p^{m}}$. Let $\hat{K}_{\infty}^{\mathrm{pf}}$ denote the $p$-adic completion of $K_{\infty}^{\mathrm{pf}}$. These fields $K_{\infty}^{(\mathrm{pf})}, K_{\infty}^{\mathrm{pf}}$ and $\hat{K}_{\infty}^{\mathrm{pf}}$ depend on the choice of a lifting of a $p$-basis of $k$ in $\mathscr{O}_{K}$. Then, we have the following inclusions

$$
K_{\infty}^{(\mathrm{pf})} \subset K_{\infty}^{\mathrm{pf}} \subset \hat{K}_{\infty}^{\mathrm{pf}}
$$

Let $H$ denote the kernel of the cyclotomic character $\chi: G_{K^{\mathrm{pf}}} \rightarrow \mathbb{Z}_{p}^{*}$. Then, the Galois group $H$ is isomorphic to the subgroup $\operatorname{Gal}\left(\bar{K} / K_{\infty}^{(\text {pf })}\right)$ of $G_{K}$. Define $\Gamma_{K}=G_{K} / H$. Let $\Gamma_{0}$ denote the subgroup $\operatorname{Gal}\left(K_{\infty}^{(\mathrm{pf})} / K^{(\mathrm{pf})}\right)\left(\simeq G_{K^{\mathrm{pf}}} / H\right)$ of $\Gamma_{K}$. Let $\Gamma_{i}(1 \leq i \leq e)$ be the subgroup of $\Gamma_{K}$ such that actions of $\beta_{i} \in \Gamma_{i}(1 \leq i \leq e)$ satisfy $\beta_{i}\left(\zeta_{p^{m}}\right)=\zeta_{p^{m}}$ and $\beta_{i}\left(b_{j}^{1 / p^{m}}\right)=b_{j}^{1 / p^{m}}$ $(i \neq j)$ and define the homomorphism $c_{i}: \Gamma_{i} \rightarrow \mathbb{Z}_{p}$ such that we have $\beta_{i}\left(b_{i}^{1 / p^{m}}\right)=b_{i}^{1 / p^{m}} \zeta_{p^{m}}^{c_{i}\left(\beta_{i}\right)}$. Then, the homomorphism $c_{i}$ defines an isomorphism $\Gamma_{i} \simeq \mathbb{Z}_{p}$ of profinite groups. With this, we can see that there exist isomorphisms of profinite groups

$$
\Gamma_{K} \simeq \Gamma_{0} \ltimes\left(\oplus_{i=1}^{e} \Gamma_{i}\right) \simeq \Gamma_{0} \ltimes \mathbb{Z}_{p}^{\oplus e} .
$$

\subsection{Definitions of $p$-adic differential modules}

We shall review the definitions of $p$-adic differential modules and have the following diagram, for a $p$-adic representation $V$ of $G_{K}$,

$$
\begin{array}{ccc}
\left(B_{\mathrm{dR}, K}^{+} \otimes_{\mathbb{Q}_{p}} V\right)^{H} & \stackrel{\theta_{K}}{\rightarrow}\left(\mathbb{C}_{p} \otimes_{\mathbb{Q}_{p}} V\right)^{H} \\
\cup & & \cup \\
D_{\mathrm{dif}}^{+}(V) & \rightarrow & D_{\mathrm{Sen}}(V) \\
\cup & & \cup \\
D_{e \text {-dif }}^{+}(V) & \rightarrow & D_{\mathrm{Bri}}(V) .
\end{array}
$$

3.1.1. The module $D_{\mathrm{Sen}}(V)$. - In the article [10], Sen shows that, for a $p$-adic representation $V$ of $G_{K^{\mathrm{pf}}}$, the $\hat{K}_{\infty}^{\mathrm{pf}}$-vector space $\left(\mathbb{C}_{p} \otimes \mathbb{Q}_{p} V\right)^{H}$ has dimension $d=\operatorname{dim}_{\mathbb{Q}_{p}} V$ and the union of the finite dimensional $K_{\infty}^{\mathrm{pf}}$-subspaces of $\left(\mathbb{C}_{p} \otimes_{\mathbb{Q}_{p}} V\right)^{H}$ stable under $\Gamma_{0}$ $\left(\simeq G_{K^{\mathrm{pf}}} / H\right)$ is a $K_{\infty}^{\mathrm{pf}}$-vector space of dimension $d$ stable under $\Gamma_{0}$ (called $\left.D_{\text {Sen }}(V)\right)$. We have $\mathbb{C}_{p} \otimes_{K_{\infty}^{\mathrm{pf}}} D_{\mathrm{Sen}}(V)=\mathbb{C}_{p} \otimes_{\mathbb{Q}_{p}} V$ and the natural map $\hat{K}_{\infty}^{\mathrm{pf}} \otimes_{K_{\infty}^{\mathrm{pf}}} D_{\mathrm{Sen}}(V) \rightarrow\left(\mathbb{C}_{p} \otimes_{\mathbb{Q}_{p}} V\right)^{H}$ is an isomorphism. Furthermore, if $\gamma \in \Gamma_{0}$ is close enough to 1 , then the series of operators on $D_{\text {Sen }}(V)$

$$
\frac{\log (\gamma)}{\log (\chi(\gamma))}=-\frac{1}{\log (\chi(\gamma))} \sum_{k \geq 1} \frac{(1-\gamma)^{k}}{k}
$$

converges to a $K_{\infty}^{\mathrm{pf}}$-linear derivation $\nabla^{(0)}: D_{\mathrm{Sen}}(V) \rightarrow D_{\mathrm{Sen}}(V)$ and does not depend on the choice of $\gamma$.

3.1.2. The module $D_{\mathrm{Bri}}(V)$. - In the article [2], Brinon generalizes Sen's work above. For a $p$-adic representation $V$ of $G_{K}$, he shows that the union of the finite dimensional $K_{\infty}^{(\mathrm{pf})}$ subspaces of $\left(\mathbb{C}_{p} \otimes_{\mathbb{Q}_{p}} V\right)^{H}$ stable under $\Gamma_{K}$ is a $K_{\infty}^{(\mathrm{pf})}$-vector space of dimension $d$ stable under $\Gamma_{K}$ (we call it $D_{\mathrm{Bri}}(V)$ ). We have $\mathbb{C}_{p} \otimes_{K_{\infty}^{(\mathrm{pf})}} D_{\mathrm{Bri}}(V)=\mathbb{C}_{p} \otimes_{\mathbb{Q}_{p}} V$ and the natural $\operatorname{map} \hat{K}_{\infty}^{\mathrm{pf}} \otimes_{K_{\infty}^{\text {(p) }}} D_{\mathrm{Bri}}(V) \rightarrow\left(\mathbb{C}_{p} \otimes_{\mathbb{Q}_{p}} V\right)^{H}$ is an isomorphism. As in the case of $D_{\mathrm{Sen}}(V)$, the $K_{\infty}^{(\mathrm{pf})}$-vector space $D_{\mathrm{Bri}}(V)$ is endowed with the action of the $K_{\infty}^{(\mathrm{pf})}$-linear derivation 
$\nabla^{(0)}=\frac{\log (\gamma)}{\log (\chi(\gamma))}$ if $\gamma \in \Gamma_{0}$ is close enough to 1 . In addition to this operator $\nabla^{(0)}$, if $\beta_{i} \in \Gamma_{i}$ is close enough to 1 , then the series of operators on $D_{\mathrm{Bri}}(V)$

$$
\frac{\log \left(\beta_{i}\right)}{c_{i}\left(\beta_{i}\right)}=-\frac{1}{c_{i}\left(\beta_{i}\right)} \sum_{k \geq 1} \frac{\left(1-\beta_{i}\right)^{k}}{k}
$$

converges to a $K_{\infty}^{(\mathrm{pf})}$-linear derivation $\nabla^{(i)}: D_{\mathrm{Bri}}(V) \rightarrow D_{\mathrm{Bri}}(V)$ and does not depend on the choice of $\beta_{i}$.

3.1.3. The module $D_{e \text {-dif }}^{+}(V)$. - In the article [1], Andreatta and Brinon generalize Fontaine's work [6]. For a $p$-adic representation $V$ of $G_{K}$, they show that the union of $K_{\infty}^{(\mathrm{pf})}\left[\left[t, t_{1}, \ldots, t_{e}\right]\right]$-submodules of finite type of $\left(B_{\mathrm{dR}, K}^{+} \otimes_{\mathbb{Q}_{p}} V\right)^{H}$ stable under $\Gamma_{K}$ is a free $K_{\infty}^{(\mathrm{pf})}\left[\left[t, t_{1}, \ldots, t_{e}\right]\right]$-module of rank d stable under $\Gamma_{K}$ (we call it $D_{e \text {-dif }}^{+}(V)$ ). We have $B_{\mathrm{dR}, K}^{+} \otimes_{K_{\infty}^{(\mathrm{pf})}\left[\left[t, t_{1}, \ldots, t_{e}\right]\right]} D_{e-\mathrm{dif}}^{+}(V)=B_{\mathrm{dR}, K}^{+} \otimes_{\mathbb{Q}_{p}} V$ and the natural map

$$
\left(B_{\mathrm{dR}, K}^{+}\right)^{H} \otimes_{K_{\infty}^{(\mathrm{pf})}\left[\left[t, t_{1}, \ldots, t_{e}\right]\right]} D_{e-\text { dif }}^{+}(V) \rightarrow\left(B_{\mathrm{dR}, K}^{+} \otimes_{\mathbb{Q}_{p}} V\right)^{H}
$$

is an isomorphism. The $K_{\infty}^{(\mathrm{pf})}\left[\left[t, t_{1}, \ldots, t_{e}\right]\right]$-module $D_{e \text {-dif }}^{+}(V)$ is endowed with the action of the $K_{\infty}^{(\text {pf })}$-linear derivations $\nabla^{(0)}=\frac{\log (\gamma)}{\log (\chi(\gamma))}$ if $\gamma \in \Gamma_{0}$ is close enough to 1 and $\nabla^{(i)}=\frac{\log \left(\beta_{i}\right)}{c_{i}\left(\beta_{i}\right)}(1 \leq i \leq e)$ if $\beta_{i} \in \Gamma_{i}$ is close enough to 1.

3.1.4. The module $D_{\mathrm{dif}}^{+}(V)$. - For a $p$-adic representation $V$ of $G_{K}$, define $D_{\mathrm{dif}}^{+}(V)$ to be ${\underset{\lim }{r}}_{r}\left(K_{\infty}^{\mathrm{pf}}\left[\left[t, t_{1}, \ldots, t_{e}\right]\right] \otimes_{K_{\infty}^{(\mathrm{pf})}\left[\left[t, t_{1}, \ldots, t_{e}\right]\right]} D_{e \text {-dif }}^{+,(r)}(V)\right)$ where we put $D_{e \text {-dif }}^{+,(r)}(V)=$ $D_{e \text {-dif }}^{+}(V) /\left(t, t_{1}, \ldots, t_{e}\right)^{r} D_{e \text {-dif }}^{+}(V)$. One can verify that $D_{\text {dif }}^{+}(V)$ is the union of $K_{\infty}^{\mathrm{pf}}\left[\left[t, t_{1}, \ldots, t_{e}\right]\right]$-submodules of finite type of $\left(B_{\mathrm{dR}, K}^{+} \otimes_{\mathbb{Q}_{p}} V\right)^{H}$ stable under $\Gamma_{0}\left(\simeq G_{K^{\mathrm{pf}}} / H\right)$ and is a free $K_{\infty}^{\mathrm{pf}}\left[\left[t, t_{1}, \ldots, t_{e}\right]\right]$-module of rank $d$ stable under $\Gamma_{0}$. Furthermore, we have $B_{\mathrm{dR}, K}^{+} \otimes_{K_{\infty}^{\mathrm{pf}}\left[\left[t, t_{1}, \ldots, t_{e}\right]\right]} D_{\mathrm{dif}}^{+}(V)=B_{\mathrm{dR}, K}^{+} \otimes_{\mathbb{Q}_{p}} V$ and the natural map $\left(B_{\mathrm{dR}, K}^{+}\right)^{H}$ $\otimes_{K_{\infty}^{\mathrm{pf}}\left[\left[t, t_{1}, \ldots, t_{e}\right]\right]} D_{\mathrm{dif}}^{+}(V) \rightarrow\left(B_{\mathrm{dR}, K}^{+} \otimes_{\mathbb{Q}_{p}} V\right)^{H}$ is an isomorphism. As in the case of $D_{e \text {-dif }}^{+}(V)$, the $K_{\infty}^{\mathrm{pf}}\left[\left[t, t_{1}, \ldots, t_{e}\right]\right]$-module $D_{\text {dif }}^{+}(V)$ is endowed with the action of the $K_{\infty}^{\mathrm{pf}}$-linear derivation $\nabla^{(0)}=\frac{\log (\gamma)}{\log (\chi(\gamma))}$ if $\gamma \in \Gamma_{0}$ is close enough to 1 .

REMARK 3.1. - $\quad$ 1. The preceding results in Subsection 3.1.1 are obtained when $V$ is a $p$-adic representation of $G_{L}=\operatorname{Gal}(\bar{L} / L)$ where $L$ is a complete discrete valuation field of characteristic 0 with perfect residue field of characteristic $p>0$ and we choose an algebraic closure $\bar{L}$ of $L$. However, in Subsection 3.1.1, for simplicity, we stated the results in the case $L=K^{\mathrm{pf}}$.

2. Note that, though many people denote the $p$-adic differential module constructed by Fontaine in [6] by $D_{\text {dif }}^{+}(V)$, the module $D_{\text {dif }}^{+}(V)$ in Subsection 3.1.4 is a little different from this module.

\subsection{Some properties of differential operators}

We shall describe the action of derivations $\left\{\nabla^{(i)}\right\}_{i=0}^{e}$ on $D_{\mathrm{Bri}}(V)$ and $D_{e \text {-dif }}^{+}(V)$. First, by a standard argument, we can show that, if $x \in D_{\mathrm{Bri}}(V)$ (resp. $D_{e \text {-dif }}^{+}(V)$ ), we have

$$
\nabla^{(0)}(x)=\lim _{\gamma \rightarrow 1} \frac{\gamma(x)-x}{\chi(\gamma)-1} \quad \text { and } \quad \nabla^{(i)}(x)=\lim _{\beta_{i} \rightarrow 1} \frac{\beta_{i}(x)-x}{c_{i}\left(\beta_{i}\right)}
$$


With this, we can easily describe the actions of $K_{\infty}^{(\mathrm{pf})}$-linear derivations $\left\{\nabla^{(i)}\right\}_{i=0}^{e}$ on $K_{\infty}^{(\mathrm{pf})}\left[\left[t, t_{1}, \ldots, t_{e}\right]\right]=D_{e \text {-dif }}^{+}\left(\mathbb{Q}_{p}\right)$ where $\mathbb{Q}_{p}$ is equipped with the structure of $p$-adic representations of $G_{K}$ induced by the trivial action of $G_{K}$.

Lemma 3.2. - The actions of $K_{\infty}^{(\mathrm{pf})}$-linear derivations $\left\{\nabla^{(i)}\right\}_{i=0}^{e}$ on $K_{\infty}^{(\mathrm{pf})}\left[\left[t, t_{1}, \ldots, t_{e}\right]\right]$ are given by $\nabla^{(0)}=t \frac{d}{d t}$ and $\nabla^{(i)}=t \frac{d}{d t_{i}}(1 \leq i \leq e)$.

Proof. - Since $\left\{\nabla^{(j)}\right\}_{j=0}^{e}$ are $K_{\infty}^{(\mathrm{pf})}$-linear derivations and we can see that we have $\nabla^{(j)}\left(t_{k}\right)=0(j \neq k)$ and $\nabla^{(i)}(t)=0(i \neq 0)$, it suffices to show that we have $\nabla^{(0)}(t)=t$ and $\nabla^{(i)}\left(t_{i}\right)=t$. These follow from

$$
\begin{aligned}
& \nabla^{(0)}(t)=\lim _{\gamma \rightarrow 1} \frac{\gamma(t)-t}{\chi(\gamma)-1}=\lim _{\gamma \rightarrow 1} \frac{\chi(\gamma) t-t}{\chi(\gamma)-1}=t \\
& \nabla^{(i)}\left(t_{i}\right)=\lim _{\beta_{i} \rightarrow 1} \frac{\beta_{i}\left(t_{i}\right)-t_{i}}{c_{i}\left(\beta_{i}\right)}=\lim _{\beta_{i} \rightarrow 1} \frac{\left(t_{i}+c_{i}\left(\beta_{i}\right) t\right)-t_{i}}{c_{i}\left(\beta_{i}\right)}=t .
\end{aligned}
$$

We extend naturally actions of $K_{\infty}^{(\mathrm{pf})}$-linear derivations $\left\{\nabla^{(i)}\right\}_{i=0}^{e}$ on $K_{\infty}^{(\mathrm{pf})}\left[\left[t, t_{1}, \ldots, t_{e}\right]\right]$ to $K_{\infty}^{(\mathrm{pf})}\left[\left[t, t_{1}, \ldots, t_{e}\right]\right]\left[t^{-1}\right]\left(\subset B_{\mathrm{dR}, K}\right)$ by putting $\nabla^{(0)}\left(t^{-1}\right)=-t^{-1}$ and $\nabla^{(i)}\left(t^{-1}\right)=0$ $(1 \leq i \leq e)$. Now, we compute the bracket [ , ] of derivations $\left\{\nabla^{(i)}\right\}_{i=0}^{e}$ on $D_{\mathrm{Bri}}(V)$ (resp. $\left.D_{e-\text { dif }}^{+}(V)\right)$.

Proposition 3.3. - On the p-adic differential module $D_{\mathrm{Bri}}(V)$ (resp. $D_{e-\mathrm{dif}}^{+}(V)$ ), we have $\left[\nabla^{(0)}, \nabla^{(i)}\right]=\nabla^{(i)}(i \neq 0)$ and $\left[\nabla^{(i)}, \nabla^{(j)}\right]=0(i, j \neq 0)$.

Proof. - The second equality follows from the commutativity of $\beta_{i}$ and $\beta_{j}$. For the first equality, we have the relation $\gamma \beta_{i}=\beta_{i}^{\chi(\gamma)} \gamma$. Then, since we have

$$
\lim _{h \rightarrow 0} \frac{a^{h+1}-a}{(h+1)-1}=a \log (a)
$$

we obtain

$$
\begin{aligned}
{\left[\nabla^{(0)}, \nabla^{(i)}\right](*) } & =\lim _{\gamma \rightarrow 1} \frac{\gamma-1}{\chi(\gamma)-1} \lim _{\beta_{i} \rightarrow 1} \frac{\beta_{i}-1}{c_{i}\left(\beta_{i}\right)}(*)-\lim _{\beta_{i} \rightarrow 1} \frac{\beta_{i}-1}{c_{i}\left(\beta_{i}\right)} \lim _{\gamma \rightarrow 1} \frac{\gamma-1}{\chi(\gamma)-1}(*) \\
& =\lim _{\beta_{i} \rightarrow 1} \lim _{\gamma \rightarrow 1} \frac{\gamma \beta_{i}-\gamma-\beta_{i}+1}{(\chi(\gamma)-1) c_{i}\left(\beta_{i}\right)}(*)-\lim _{\beta_{i} \rightarrow 1} \lim _{\gamma \rightarrow 1} \frac{\beta_{i} \gamma-\gamma-\beta_{i}+1}{(\chi(\gamma)-1) c_{i}\left(\beta_{i}\right)}(*) \\
& =\lim _{\beta_{i} \rightarrow 1} \lim _{\gamma \rightarrow 1} \frac{\beta_{i}^{\chi(\gamma)} \gamma-\beta_{i} \gamma}{(\chi(\gamma)-1) c_{i}\left(\beta_{i}\right)}(*) \\
& =\lim _{\beta_{i} \rightarrow 1} \frac{\beta_{i} \log \left(\beta_{i}\right)}{c_{i}\left(\beta_{i}\right)}(*) \\
& =\nabla^{(i)}(*) .
\end{aligned}
$$

Proposition 3.4. - The action of the $K_{\infty}^{(\mathrm{pf})}$-linear derivation $\nabla^{(i)}(i \neq 0)$ on $D_{\mathrm{Bri}}(V)$ is nilpotent.

Proof. - From the equality $\nabla^{(0)} \nabla^{(i)}-\nabla^{(i)} \nabla^{(0)}=\nabla^{(i)}$, we get $\nabla^{(0)}\left(\nabla^{(i)}\right)^{r}-\left(\nabla^{(i)}\right)^{r} \nabla^{(0)}$ $=r\left(\nabla^{(i)}\right)^{r}$ and $\operatorname{tr}\left(r\left(\nabla^{(i)}\right)^{r}\right)=0$ for all $r \in \mathbb{N}$. Since the characteristic of $K_{\infty}^{(\mathrm{pf})}$ is 0 , we obtain $\operatorname{tr}\left(\left(\nabla^{(i)}\right)^{r}\right)=0$ for all $r \in \mathbb{N}$. As is well known in linear algebra, this shows that the action of the $K_{\infty}^{(\mathrm{pf})}$-linear derivation $\nabla^{(i)}(i \neq 0)$ on $D_{\mathrm{Bri}}(V)$ is nilpotent. 
Notation . - For simplicity, put

$$
R=K_{\infty}^{(\mathrm{pf})}\left[t, \frac{t_{1}}{t}, \ldots, \frac{t_{e}}{t}\right] \quad \text { or } \quad K_{\infty}^{(\mathrm{pf})}\left[\left[t, t_{1}, \ldots, t_{e}\right]\right] .
$$

Proposition 3.5. - Let $M$ be a finitely generated free $R[1 / t]$-module endowed with $K_{\infty}^{(\mathrm{pf})}$-linear derivations $\left\{\nabla^{(i)}\right\}_{i=0}^{e}$ which satisfy the same properties in Lemma 3.2 and Proposition 3.3. Assume that we can choose a basis $\left\{g_{j}\right\}_{j=1}^{d}$ of $M$ over $R[1 / t]$ such that $\nabla^{(0)}\left(g_{j}\right)=0$. Then, the action of $\nabla^{(i)}(i \neq 0)$ on this basis is given by $\nabla^{(i)}\left(g_{j}\right)=t \sum_{k=1}^{d} c_{k} g_{k}$ where $c_{k}$ is an element of $R$ such that $\nabla^{(0)}\left(c_{k}\right)=0$.

Proof. - Since $\left\{g_{j}\right\}_{j=1}^{d}$ forms a basis of $M$ over $R[1 / t]$, we can write, for $i \neq 0$,

$$
\nabla^{(i)}\left(g_{j}\right)=\sum_{k=1}^{d} a_{k} g_{k} \quad\left(a_{k} \in R[1 / t]\right) .
$$

Then, the relation $\left[\nabla^{(0)}, \nabla^{(i)}\right]=\nabla^{(i)}(i \neq 0)$ of Proposition 3.3 says that we have $\sum_{k=1}^{d} \nabla^{(0)}\left(a_{k}\right) g_{k}=\sum_{k=1}^{d} a_{k} g_{k}$. Note that we have $\nabla^{(0)}\left(g_{j}\right)=0$ by hypothesis. Hence, we obtain the differential equation $\nabla^{(0)}\left(a_{k}\right)=a_{k}$. Define an element $c_{k}$ of $R[1 / t]$ to be $a_{k} / t$. Then, we can see that $c_{k}$ satisfies $\nabla^{(0)}\left(c_{k}\right)=a_{k} / t-a_{k} / t=0$ and that $c_{k}$ is contained in $R$. Thus, the solution of the differential equation $\nabla^{(0)}\left(a_{k}\right)=a_{k}$ in $\mathrm{R}[1 / \mathrm{t}]$ has the following form

$$
a_{k}=c_{k} t
$$

where $c_{k}$ is an element of $R$ such that $\nabla^{(0)}\left(c_{k}\right)=0$. Hence, from (3.1) and (3.2), we obtain, for $i \neq 0, \nabla^{(i)}\left(g_{j}\right)=t \sum_{k=1}^{d} c_{k} g_{k}$ where $c_{k}$ is an element of $R$ such that $\nabla^{(0)}\left(c_{k}\right)=0$.

Corollary 3.6. - With notations as in Proposition 3.5 above, we have the following presentation

$$
\left(\nabla^{(1)}\right)^{k_{1}} \cdots\left(\nabla^{(e)}\right)^{k_{e}}\left(g_{j}\right)=t^{k_{1}+\cdots+k_{e}} \sum_{k=1}^{d} c_{k} g_{k}
$$

where $c_{k}$ is an element of $R$ such that $\nabla^{(0)}\left(c_{k}\right)=0$.

\section{Proof of the main theorem}

In this section, we keep the notation and the assumption of Section 3.

\subsection{Main theorem for Hodge-Tate representations}

Proposition 4.1 ([10, Section (2.3)]). - If $V$ is a Hodge-Tate representation of $G_{K^{\mathrm{p}}}$, there exists a $\Gamma_{0}$-equivariant isomorphism of $K_{\infty}^{\mathrm{pf}}$-vector spaces

$$
D_{\mathrm{Sen}}(V) \simeq \bigoplus_{j=1}^{d=\operatorname{dim}_{\mathbb{Q}_{p}} V} K_{\infty}^{\mathrm{pf}}\left(n_{j}\right) \quad\left(n_{j} \in \mathbb{Z}\right)
$$


REMARK 4.2. - In general, if $L$ denotes a complete discrete valuation field of characteristic 0 with perfect residue field of characteristic $p>0$ and $V$ is a Hodge-Tate representation of $G_{L}=\operatorname{Gal}(\bar{L} / L)$ where we choose an algebraic closure $\bar{L}$ of $L$, Sen shows that there exists a $G_{L} / H$-equivariant isomorphism of $L_{\infty}\left(=\cup_{m \geq 1} L\left(\zeta_{p^{m}}\right)\right)$-vector spaces ([10, Section (2.3)])

$$
D_{\text {Sen }}(V) \simeq \bigoplus_{j=1}^{d=\operatorname{dim}_{\mathbb{Q}_{p}} V} L_{\infty}\left(n_{j}\right) \quad\left(n_{j} \in \mathbb{Z}\right) .
$$

COROLlary 4.3. - For a p-adic representation $V$ of $G_{K}$, assume that $V$ is a Hodge-Tate representation of $G_{K^{\mathrm{pf}}}$. Then, there exists a $\nabla^{(0)}$ - equivariant isomorphism of $K_{\infty}^{(\mathrm{pf})}$-vector spaces

$$
D_{\mathrm{Bri}}(V) \simeq \nabla^{(0)} \quad \bigoplus_{j=1}^{d=\operatorname{dim}_{\mathbb{Q}_{p}} V} K_{\infty}^{(\mathrm{pf})}\left(n_{j}\right) \quad\left(n_{j} \in \mathbb{Z}\right) .
$$

Here, $\simeq_{\nabla^{(0)}}$ denotes $a \nabla^{(0)}$-equivariant isomorphism. Furthermore, the multiplicity of $\left\{n_{j}\right\}_{j=1}^{d}$ is the same as that of $\left\{n_{j}\right\}_{j=1}^{d}$ in Proposition 4.1.

Proof. - From the presentation of Proposition 4.1, the action of the $K_{\infty}^{\mathrm{pf}}$-linear derivation $\nabla^{(0)}$ on $D_{\text {Sen }}(V)$ is semi-simple and its eigenvalues are integers. Thus, the action of the $K_{\infty}^{(\mathrm{pf})}$-linear derivation $\nabla^{(0)}$ on the subspace $D_{\mathrm{Bri}}(V)$ of $D_{\mathrm{Sen}}(V)$ is also semi-simple and its eigenvalues are the same. Therefore, we obtain a $\nabla^{(0)}$-equivariant isomorphism $D_{\mathrm{Bri}}(V) \simeq_{\nabla^{(0)}} \bigoplus_{j=1}^{d} K_{\infty}^{(\mathrm{pf})}\left(n_{j}\right)\left(n_{j} \in \mathbb{Z}\right)$. By tensoring $K_{\infty}^{\mathrm{pf}} \otimes_{K_{\infty}^{(\mathrm{pf})}}$ over both sides, we obtain $K_{\infty}^{\mathrm{pf}} \otimes_{K_{\infty}^{(\mathrm{pf})}} D_{\mathrm{Bri}}(V) \simeq_{\nabla^{(0)}} \bigoplus_{j=1}^{d} K_{\infty}^{\mathrm{pf}}\left(n_{j}\right)\left(n_{j} \in \mathbb{Z}\right)$. Furthermore, since we have $K_{\infty}^{\mathrm{pf}} \otimes_{K_{\infty}^{(\mathrm{pf})}} D_{\mathrm{Bri}}(V) \hookrightarrow D_{\mathrm{Sen}}(V)$ by definition and both sides have the same dimension $d$ over $K_{\infty}^{\mathrm{pf}}$, we obtain $K_{\infty}^{\mathrm{pf}} \otimes_{K_{\infty}^{(\mathrm{pf})}} D_{\mathrm{Bri}}(V)=D_{\mathrm{Sen}}(V)$ and can see that the multiplicity of $\left\{n_{j}\right\}_{j=1}^{d}$ is the same as that of $\left\{n_{j}\right\}_{j=1}^{d}$ in Proposition 4.1.

Theorem 4.4. - Let $K$ be a complete discrete valuation field of characteristic 0 with residue field $k$ of characteristic $p>0$ such that $\left[k: k^{p}\right]=p^{e}<+\infty$ and $V$ be a p-adic representation of $G_{K}$. Let $K^{\mathrm{pf}}$ be the field extension of $K$ defined as before. Then, $V$ is a Hodge-Tate representation of $G_{K}$ if and only if $V$ is a Hodge-Tate representation of $G_{K^{\mathrm{p}}}$.

Proof. - We shall prove the main theorem in two parts.

(1) $V: H T$ rep. of $G_{K} \Rightarrow V: H T$ rep. of $G_{K^{\mathrm{pf}}}$. - Since $V$ is a Hodge-Tate representation of $G_{K}$, there exists a $G_{K}$-equivariant isomorphism of $B_{\mathrm{HT}, K}$-modules

$$
B_{\mathrm{HT}, K} \otimes_{\mathbb{Q}_{p}} V \simeq\left(B_{\mathrm{HT}, K}\right)^{d=\operatorname{dim}_{\mathbb{Q}_{p}} V .}
$$

Now, by tensoring $B_{\mathrm{HT}, K^{\mathrm{pf}}} \otimes_{B_{\mathrm{HT}, K}}$ (which is induced by the $G_{K^{\mathrm{pf}}}$-equivariant surjection $\left.p: B_{\mathrm{HT}, K} \rightarrow B_{\mathrm{HT}, K^{\mathrm{pf}}}: t_{i} / t \mapsto 0\right)$ over (4.1), we obtain a $G_{K^{\mathrm{pf}}}$-equivariant isomorphism of $B_{\mathrm{HT}, K^{\mathrm{pf}}}-$ modules

$$
B_{\mathrm{HT}, K^{\mathrm{pf}}} \otimes_{\mathbb{Q}_{p}} V \simeq\left(B_{\mathrm{HT}, K^{\mathrm{pf}}}\right)^{d} .
$$

This means that $V$ is a Hodge-Tate representation of $G_{K^{\mathrm{p}}}$. 
(2) $V: H T$ rep. of $G_{K^{\mathrm{pf}}} \Rightarrow V: H T$ rep. of $G_{K}$. - For simplicity, put $R=K_{\infty}^{(\mathrm{pf})}\left[t, \frac{t_{1}}{t}, \ldots, \frac{t_{e}}{t}\right]$. We shall construct the $K_{\infty}^{(\mathrm{pf})}$-linearly independent elements $\left\{f_{j}^{(*)}\right\}_{j=1}^{d=\operatorname{dim}_{\mathbb{Q}_{p}} V}$

of $R[1 / t] \otimes_{K_{\infty}^{(\mathrm{pf})}} D_{\mathrm{Bri}}(V)\left(\subset B_{\mathrm{HT}, K} \otimes_{\mathbb{Q}_{p}} V\right)$ such that $\nabla^{(i)}\left(f_{j}^{(*)}\right)=0$ for all $0 \leq i \leq e$ and $1 \leq j \stackrel{\infty}{\leq} d$.

(A) Construction of $\left\{f_{j}^{(*)}\right\}_{j=1}^{d} \in R[1 / t] \otimes_{K_{\infty}^{(\mathrm{pf})}} D_{\mathrm{Bri}}(V)$. - From the presentation of Corollary 4.3 above, if we twist by some powers of $t$, we obtain a basis $\left\{f_{j}\right\}_{j=1}^{d}$ of $R[1 / t] \otimes_{K_{\infty}^{(\mathrm{pr})}} D_{\mathrm{Bri}}(V)$ over $R[1 / t]$ such that $\nabla^{(0)}\left(f_{j}\right)=0$ for all $1 \leq j \leq d$. Thus, by applying Corollary 3.6 to the $R[1 / t]$-module $R[1 / t] \otimes_{K_{\infty}^{(\mathrm{pf})}} D_{\mathrm{Bri}}(V)$ generated by $\left\{f_{j}\right\}_{j=1}^{d}$, we can deduce

$$
\left(\nabla^{(1)}\right)^{k_{1}} \cdots\left(\nabla^{(e)}\right)^{k_{e}}\left(f_{j}\right)=t^{k_{1}+\cdots+k_{e}} \sum_{k=1}^{d} c_{k} f_{k}
$$

where $c_{k}$ is an element of $R$ such that $\nabla^{(0)}\left(c_{k}\right)=0$. Furthermore, since the action of $K_{\infty}^{(\mathrm{pf})}$-linear derivation $\nabla^{(i)}(i \neq 0)$ on $D_{\mathrm{Bri}}(V)$ is nilpotent by Proposition 3.4, if we take $n \in \mathbb{N}$ large enough, we obtain

$$
\left(\nabla^{(i)}\right)^{n}\left(f_{j}\right)=0 \text { for all } 1 \leq j \leq d \text { and } 1 \leq i \leq e .
$$

Define an element $f_{j}^{(*)}$ of $R[1 / t] \otimes_{K_{\infty}^{(\mathrm{pf})}} D_{\mathrm{Bri}}(V)$ by

$$
f_{j}^{(*)}=\sum_{0 \leq k_{1}, \ldots, k_{e}}(-1)^{k_{1}+\cdots+k_{e}} \frac{t_{1}^{k_{1}} \cdots t_{e}^{k_{e}}}{k_{1} ! \cdots k_{e} ! t^{k_{1}+\cdots+k_{e}}}\left(\nabla^{(1)}\right)^{k_{1}} \cdots\left(\nabla^{(e)}\right)^{k_{e}}\left(f_{j}\right) .
$$

Note that this series is a finite sum by (4.3) and thus $f_{j}^{(*)}$ actually defines an element of $R[1 / t] \otimes_{K_{\infty}^{(\mathrm{pr})}} D_{\mathrm{Bri}}(V)$. Then, it follows easily that we have $\nabla^{(i)}\left(f_{j}^{(*)}\right)=0$ for all $1 \leq i \leq e$ and $1 \leq j \leq d$ by using the Leibniz rule. Furthermore, by using $(4.2)$ and the fact $\nabla^{(0)}\left(f_{j}\right)=0$, we can deduce that we have $\nabla^{(0)}\left(f_{j}^{(*)}\right)=0$ for all $1 \leq j \leq d$.

(B) $\left\{f_{j}^{(*)}\right\}_{j=1}^{d} \in R[1 / t] \otimes_{K_{\infty}^{(\mathrm{pf})}} D_{\mathrm{Bri}}(V)$ is linearly independent over $K_{\infty}^{(\mathrm{pf})}$. - By the presentation of $f_{j}^{(*)}$, we have

$$
f_{j}^{(*)}=f_{j}+g_{j} \quad\left(g_{j} \in\left(\frac{t_{1}}{t}, \ldots, \frac{t_{e}}{t}\right)\left(B_{\mathrm{HT}, K} \otimes_{\mathbb{Q}_{p}} V\right)\right) .
$$

Since $\left\{f_{j}\right\}_{j=1}^{d}$ forms a basis of $R[1 / t] \otimes_{K_{\infty}^{(\mathrm{pf})}} D_{\mathrm{Bri}}(V)$ over $R[1 / t]$, it is, in particular, linearly independent over $K_{\infty}^{(\mathrm{pf})}(\subset R[1 / t])$. Thus, $\left\{\overline{f_{j}}={\overline{f_{j}}}^{(*)}\right\}_{j=1}^{d}$ (-denotes the reduction modulo $\left.\left(t_{1}, \ldots, t_{e}\right)\right)$ is linearly independent over $K_{\infty}^{(\mathrm{pf})}$ and we can see that $\left\{f_{j}^{(*)}\right\}_{j=1}^{d}$ is linearly independent over $K_{\infty}^{(\mathrm{pf})}$ in $R[1 / t] \otimes_{K_{\infty}^{(\mathrm{pf})}} D_{\mathrm{Bri}}(V)$.

(C) Conclusion. - Therefore, on the $K$-vector space generated by $\left\{f_{j}^{(*)}\right\}_{j=1}^{d}, \log (\gamma)$ and $\left\{\log \left(\beta_{i}\right)\right\}_{i=1}^{e}$ act trivially $\left(\Leftrightarrow \nabla^{(0)}\left(f_{j}^{(*)}\right)=0\right.$ and $\nabla^{(i)}\left(f_{j}^{(*)}\right)=0$ for all $1 \leq i \leq e$ and $1 \leq j \leq d)$. Thus, this means that $\Gamma_{K}$ acts on this $K$-vector space via finite quotient and there exists a finite field extension $L / K$ in $K_{\infty}^{(\mathrm{pf})}$ such that $\left\{f^{(*)}\right\}_{j=1}^{d}$ forms a basis of $D_{\mathrm{HT}, L}(V)$ 
over $L$. Since a potentially Hodge-Tate representation of $G_{K}$ is a Hodge-Tate representation of $G_{K}$, this completes the proof.

\subsection{Main theorem for de Rham representations}

Lemma 4.5. - For a p-adic representation $V$ of $G_{K}$, assume that $V$ is a de Rham representation of $G_{K^{\mathrm{pf}}}$. Then, we can choose a basis $\left\{h_{j}\right\}_{j=1}^{d=\operatorname{dim}_{\mathbb{Q}_{p}} V}$ of $D_{\mathrm{dif}}^{+}(V)[1 / t]$ over $K_{\infty}^{\mathrm{pf}}\left[\left[t, t_{1}, \ldots, t_{e}\right]\right][1 / t]$ such that the action of $\Gamma_{0}$ on $\left\{h_{j}\right\}_{j=1}^{d}$ is trivial.

Proof. - Since $V$ is a de Rham representation of $G_{K^{\text {pf }}}$, there exists a basis $\left\{h_{j}\right\}_{j=1}^{d}$ of $B_{\mathrm{dR}, K^{\mathrm{pf}}} \otimes_{\mathbb{Q}_{p}} V$ over $B_{\mathrm{dR}, K^{\mathrm{pf}}}$ such that the action of $G_{K^{\mathrm{pf}}}$ on $\left\{h_{j}\right\}_{j=1}^{d}$ is trivial. We can see that these elements $\left\{h_{j}\right\}_{j=1}^{d}$ are contained in $D_{\text {dif }}^{+}(V)[1 / t]$ by definition. For each $j$, if we twist $h_{j}$ by some power of $t$, we obtain an element $g_{j}$ of $B_{\mathrm{dR}, K^{\mathrm{pf}}}^{+} \otimes_{\mathbb{Q}_{p}} V$ such that $g_{j} \notin t B_{\mathrm{dR}, K^{\mathrm{pf}}}^{+} \otimes_{\mathbb{Q}_{p}} V$. Then, it follows that $g_{j}$ is contained in $D_{\text {dif }}^{+}(V)$ and satisfies $\overline{g_{j}} \neq 0$ (-denotes the reduction modulo $\left.\left(t, t_{1}, \ldots, t_{e}\right) D_{\text {dif }}^{+}(V)\right)$. Since $D_{\text {dif }}^{+}(V)$ is a free module of rank $d$ over the local ring $K_{\infty}^{\mathrm{pf}}\left[\left[t, t_{1}, \ldots, t_{e}\right]\right]$ and $\left\{\overline{g_{j}}\right\}_{j=1}^{d}$ forms a basis of $D_{\mathrm{Sen}}(V)$ over $K_{\infty}^{\mathrm{pf}}$, the lifting $\left\{g_{j}\right\}_{j=1}^{d}$ of $\left\{\bar{g}_{j}\right\}_{j=1}^{d}$ in $D_{\text {dif }}^{+}(V)$ forms a basis of $D_{\text {dif }}^{+}(V)$ over $K_{\infty}^{\mathrm{pf}}\left[\left[t, t_{1}, \ldots, t_{e}\right]\right]$. Thus, it follows that $\left\{h_{j}\right\}_{j=1}^{d}$ forms a basis of $D_{\text {dif }}^{+}(V)[1 / t]$ over $K_{\infty}^{\mathrm{pf}}\left[\left[t, t_{1}, \ldots, t_{e}\right]\right][1 / t]$.

With notations as above, note that, since we have the inclusion $D_{e \text {-dif }}^{+}(V) \hookrightarrow D_{\text {dif }}^{+}(V)[1 / t]$ by definition, any element $g$ of $D_{e \text {-dif }}^{+}(V)$ can be written as $g=\sum_{k=l}^{+\infty}\left(\sum_{j=1}^{d} a_{j k} h_{j}\right) t^{k}$ $\left(a_{j k} \in K_{\infty}^{\mathrm{pf}}\left[\left[t_{1}, \ldots, t_{e}\right]\right]\right)$.

Remark 4.6. - Keep the notation as in Lemma 4.5. Since we assume that $V$ is a de Rham representation of $G_{K^{\mathrm{pf}}}$, by Corollary 4.3 , there exists a basis $\left\{v_{j}\right\}_{j=1}^{d}$ of $D_{\mathrm{Bri}}(V)$ over $K_{\infty}^{(\mathrm{pf})}$ such that $\nabla^{(0)}\left(v_{j}\right)=n_{j} v_{j}$. Put $M=\operatorname{Max}\left(n_{j}\right)_{j=1}^{d}$. Then, for an element $g \in D_{e \text {-dif }}^{+}(V)$, there exists an element $\sum_{k=n}^{+\infty}\left(\sum_{j=1}^{d} c_{j k} h_{j}\right) t^{k}$ of $\left(t, t_{1}, \ldots, t_{e}\right) D_{e \text {-dif }}^{+}(V)$ such that we can write

$$
g=\sum_{k=m}^{M}\left(\sum_{j=1}^{d} b_{j k} h_{j}\right) t^{k}+\sum_{k=n}^{+\infty}\left(\sum_{j=1}^{d} c_{j k} h_{j}\right) t^{k} \quad\left(b_{j k}, c_{j k} \in K_{\infty}^{\mathrm{pf}}\left[\left[t_{1}, \ldots, t_{e}\right]\right]\right) .
$$

Thus, $g^{\prime}=\sum_{k=m}^{M}\left(\sum_{j=1}^{d} b_{j k} h_{j}\right) t^{k}$ defines an element of $D_{e \text {-dif }}^{+}(V)$.

Lemma 4.7. - With notations as above, for an element $g^{\prime}=\sum_{k=m}^{M}\left(\sum_{j=1}^{d} b_{j k} h_{j}\right) t^{k}$ of $D_{e \text {-dif }}^{+}(V)$, each $\left(\sum_{j=1}^{d} b_{j k} h_{j}\right) t^{k}$ is contained in $D_{e \text {-dif }}^{+}(V)$.

Proof. - We shall prove this lemma by induction on the smallest degree of $g^{\prime}$ with respect to $t$. Since we have $g^{\prime}-\left(\sum_{j=1}^{d} b_{j m} h_{j}\right) t^{m} \in D_{e-\mathrm{dif}}^{+}(V)$ if $\left(\sum_{j=1}^{d} b_{j m} h_{j}\right) t^{m}$ is contained in $D_{e \text {-dif }}^{+}(V)$, it suffices to show that $\left(\sum_{j=1}^{d} b_{j m} h_{j}\right) t^{m}$ is contained in $D_{e \text {-dif }}^{+}(V)$. Since the $K_{\infty}^{\mathrm{pf}}\left[\left[t_{1}, \ldots, t_{e}\right]\right]$-linear derivation $\nabla^{(0)}$ acts trivially on $\left\{h_{j}\right\}_{j=1}^{d}$, we have

$$
\prod_{k=m+1}^{M}\left(\nabla^{(0)}-k\right)\left(g^{\prime}\right)=\left(\prod_{k=m+1}^{M}(m-k)\right)\left(\sum_{j=1}^{d} b_{j m} h_{j}\right) t^{m} .
$$

It follows that $\left(\sum_{j=1}^{d} b_{j m} h_{j}\right) t^{m}$ is contained in $D_{e \text {-dif }}^{+}(V)$ since the action of $\nabla^{(0)}$ on $D_{e-\text { dif }}^{+}(V)$ is stable. Thus, this completes the proof. 
Proposition 4.8. - For a p-adic representation $V$ of $G_{K}$, assume that $V$ is a de Rham representation of $G_{K^{\mathrm{pf}}}$. Then, there exists a $\nabla^{(0)}$-equivariant isomorphism of $K_{\infty}^{(\mathrm{pf})}\left[\left[t, t_{1}, \ldots, t_{e}\right]\right]-$ modules

$$
D_{e-\text { dif }}^{+}(V) \simeq_{\nabla^{(0)}} \bigoplus_{j=1}^{d=\operatorname{dim}_{\mathbb{Q}_{p}} V} K_{\infty}^{(\mathrm{pf})}\left[\left[t, t_{1}, \ldots, t_{e}\right]\right]\left(n_{j}\right) \quad\left(n_{j} \in \mathbb{Z}\right) .
$$

Proof. - Since $V$ is also a Hodge-Tate representation of $G_{K^{\mathrm{p}}}$, by Corollary 4.3, there exists a basis $\left\{v_{j}\right\}_{j=1}^{d}$ of $D_{e \text {-dif }}^{+}(V) /\left(t, t_{1}, \ldots, t_{e}\right) D_{e \text {-dif }}^{+}(V) \simeq D_{\mathrm{Bri}}(V)$ over $K_{\infty}^{(\mathrm{pf})}$ such that it gives a $\nabla^{(0)}$-equivariant isomorphism of $K_{\infty}^{(\text {pf })}$-vector spaces

$$
D_{e-\text { dif }}^{+}(V) /\left(t, t_{1}, \ldots, t_{e}\right) D_{e-\text { dif }}^{+}(V) \simeq_{\nabla^{(0)}} \bigoplus_{j=1}^{d} K_{\infty}^{(\mathrm{pf})}\left(n_{j}\right): v_{j} \mapsto t^{n_{j}}
$$

Since $D_{e \text {-dif }}^{+}(V)$ is a free module of rank $d$ over the local ring $K_{\infty}^{(\mathrm{pf})}\left[\left[t, t_{1}, \ldots, t_{e}\right]\right]$, any lifting $\left\{g_{j}\right\}_{j=1}^{d}$ of $\left\{v_{j}\right\}_{j=1}^{d}$ in $D_{e \text {-dif }}^{+}(V)$ forms a basis of $D_{e \text {-dif }}^{+}(V)$ over $K_{\infty}^{(\mathrm{pf})}\left[\left[t, t_{1}, \ldots, t_{e}\right]\right]$. Let $\left\{h_{j}\right\}_{j=1}^{d}$ denote a basis of $D_{\text {dif }}^{+}(V)[1 / t]$ over $K_{\infty}^{\mathrm{pf}}\left[\left[t, t_{1}, \ldots, t_{e}\right]\right][1 / t]$ such that $\nabla^{(0)}\left(h_{j}\right)=0$ obtained in Lemma 4.5. Then, we may assume that each $g_{j}$ is written as $g_{j}=\sum_{k=m}^{M}\left(\sum_{l=1}^{d} b_{k l} h_{l}\right) t^{k}\left(b_{k l} \in K_{\infty}^{\mathrm{pf}}\left[\left[t_{1}, \ldots, t_{e}\right]\right]\right)$ where we take $M \in \mathbb{N}$ as in Remark 4.6. Now, define an element $f_{j}$ of $D_{e \text {-dif }}^{+}(V)$ (Lemma 4.7 above) by

$$
f_{j}=\left(\sum_{l=1}^{d} b_{n_{j} l} h_{l}\right) t^{n_{j}}
$$

It is easy to see $\nabla^{(0)}\left(f_{j}\right)=n_{j} f_{j}$. Therefore, the rest is to show that $\left\{f_{j}\right\}_{j=1}^{d}$ forms a basis of $D_{e \text {-dif }}^{+}(V)$ over $K_{\infty}^{(\mathrm{pf})}\left[\left[t, t_{1}, \ldots, t_{e}\right]\right]$. To prove that $\left\{f_{j}\right\}_{j=1}^{d}$ is a lifting of $\left\{v_{j}\right\}_{j=1}^{d}$, it suffices to show $g_{j}-f_{j} \in\left(t, t_{1}, \ldots, t_{e}\right) D_{e \text {-dif }}^{+}(V)$. For each $g_{j}$, put $s_{k}=\left(\sum_{l=1}^{d} b_{k l} h_{l}\right) t^{k} \in D_{e \text {-dif }}^{+}(V)$ (Lemma 4.7 above). Since we have $\nabla^{(0)}\left(\overline{s_{k}}\right)=k \overline{s_{k}}$ ( - denotes the reduction modulo $\left.\left(t, t_{1}, \ldots, t_{e}\right)\right)$ and this means that $\overline{s_{k}}$ is an eigenvector of $\nabla^{(0)}$, it follows that the elements $\left\{v_{j}, \overline{s_{k}} \neq 0\right\}_{k \neq n_{j}}$ are linearly independent over $K_{\infty}^{(\mathrm{pf})}$ in $D_{\mathrm{Bri}}(V)$. Since we have $v_{j}=\sum_{k=m}^{M} \overline{s_{k}}$ by definition, it follows that we obtain $\overline{s_{k}}=0$ for $k \neq n_{j}$. This means that we have $s_{k} \in\left(t, t_{1}, \ldots, t_{e}\right) D_{e \text {-dif }}^{+}(V)\left(k \neq n_{j}\right)$ and $g_{j}-f_{j} \in\left(t, t_{1}, \ldots, t_{e}\right) D_{e \text {-dif }}^{+}(V)$. Thus, this completes the proof.

REMARK 4.9. - In general, it is evident from the proof that, if $L$ denotes a complete discrete valuation field of characteristic 0 with perfect residue field of characteristic $p>0$ and $V$ is a de Rham representation of $G_{L}=\operatorname{Gal}(\bar{L} / L)$ where we choose an algebraic closure $\bar{L}$ of $L$, we have a $\nabla^{(0)}$-equivariant isomorphism of $L_{\infty}[[t]]$-modules

$$
D_{\text {dif }}^{+}(V) \simeq{ }_{\nabla^{(0)}} \bigoplus_{j=1}^{d=\operatorname{dim}_{\mathbb{Q}_{p}} V} L_{\infty}[[t]]\left(n_{j}\right) \quad\left(n_{j} \in \mathbb{Z}\right) .
$$

THeOREM 4.10. - Let $K$ be a complete discrete valuation field of characteristic 0 with residue field $k$ of characteristic $p>0$ such that $\left[k: k^{p}\right]=p^{e}<+\infty$ and $V$ be a p-adic representation of $G_{K}$. Let $K^{\mathrm{pf}}$ be the field extension of $K$ defined as before. Then, $V$ is a de Rham representation of $G_{K}$ if and only if $V$ is a de Rham representation of $G_{K^{\mathrm{pr}}}$. 
Proof. - We shall prove the main theorem in two parts.

(1) $V: d R$ rep. of $G_{K} \Rightarrow V: d R$ rep. of $G_{K^{\mathrm{pf}}}$. - Since $V$ is a de Rham representation of $G_{K}$, there exists a $G_{K}$-equivariant isomorphism of $B_{\mathrm{dR}, K}$-modules

$$
B_{\mathrm{dR}, K} \otimes_{\mathbb{Q}_{p}} V \simeq\left(B_{\mathrm{dR}, K}\right)^{d=\operatorname{dim}_{\mathbb{Q}_{p}} V} .
$$

Now, by tensoring $B_{\mathrm{dR}, K^{\mathrm{pf}}} \otimes_{B_{\mathrm{dR}, K}}$ (which is induced by the $G_{K^{\mathrm{pf}} \text {-equivariant surjection }}$ $p: B_{\mathrm{dR}, K} \rightarrow B_{\mathrm{dR}, K^{\mathrm{pf}}}: t_{i} \mapsto 0$ ) over (4.4), we obtain a $G_{K^{\mathrm{pf}}}$-equivariant isomorphism of $B_{\mathrm{dR}, K^{\mathrm{pf}}-\text { modules }}$

$$
B_{\mathrm{dR}, K^{\mathrm{pf}}} \otimes_{\mathbb{Q}_{p}} V \simeq\left(B_{\mathrm{dR}, K^{\mathrm{pf}}}\right)^{d} .
$$

This means that $V$ is a de $\mathrm{Rham}$ representation of $G_{K^{\mathrm{pf}}}$.

(2) $V: d R$ rep. of $G_{K^{\mathrm{pf}}} \Rightarrow V: d R$ rep. of $G_{K}$. - For simplicity, put $R=K_{\infty}^{(\mathrm{pf})}\left[\left[t, t_{1}, \ldots, t_{e}\right]\right]$. We shall construct the $K_{\infty}^{(\mathrm{pf})}$-linearly independent elements $\left\{f_{j}^{(*)}\right\}_{j=1}^{d=\operatorname{dim}_{\mathbb{Q}_{p}} V}$ of $R[1 / t] \otimes_{R} D_{e-\text { dif }}^{+}(V)\left(\subset B_{\mathrm{dR}, K} \otimes_{\mathbb{Q}_{p}} V\right)$ such that $\nabla^{(i)}\left(f_{j}^{(*)}\right)=0$ for all $0 \leq i \leq e$ and $1 \leq j \leq d$.

(A) Construction of $\left\{f_{j}^{(*)}\right\}_{j=1}^{d} \in R[1 / t] \otimes_{R} D_{e-\text { dif }}^{+}(V)$. - From the presentation of Proposition 4.8 above, if we twist by some powers of $t$, we obtain a basis $\left\{f_{j}\right\}_{j=1}^{d}$ of $R[1 / t] \otimes_{R} D_{e \text {-dif }}^{+}(V)$ over $R[1 / t]$ such that $\nabla^{(0)}\left(f_{j}\right)=0$ for all $1 \leq j \leq d$. Thus, by applying Corollary 3.6 to the $R[1 / t]$-module $R[1 / t] \otimes_{R} D_{e \text {-dif }}^{+}(V)$ generated by $\left\{f_{j}\right\}_{j=1}^{d}$, we can deduce

$$
\left(\nabla^{(1)}\right)^{k_{1}} \cdots\left(\nabla^{(e)}\right)^{k_{e}}\left(f_{j}\right)=t^{k_{1}+\cdots+k_{e}} \sum_{k=1}^{d} c_{k} f_{k}
$$

where $c_{k}$ is an element of $R$ such that $\nabla^{(0)}\left(c_{k}\right)=0$. Define an element $f_{j}^{(*)}$ of $R[1 / t] \otimes_{R} D_{e-\text { dif }}^{+}(V)$ by

$$
f_{j}^{(*)}=\sum_{0 \leq k_{1}, \ldots, k_{e}}(-1)^{k_{1}+\cdots+k_{e}} \frac{t_{1}^{k_{1}} \cdots t_{e}^{k_{e}}}{k_{1} ! \cdots k_{e} ! t^{k_{1}+\cdots+k_{e}}}\left(\nabla^{(1)}\right)^{k_{1}} \cdots\left(\nabla^{(e)}\right)^{k_{e}}\left(f_{j}\right) .
$$

Note that this series converges in $R[1 / t] \otimes_{R} D_{e \text {-dif }}^{+}(V)$ for $\left(t_{1}, \ldots, t_{e}\right)$-adic topology by (4.5) and thus $f_{j}^{(*)}$ actually defines an element of $R[1 / t] \otimes_{R} D_{e \text {-dif }}^{+}(V)$. Then, it follows easily that we have $\nabla^{(i)}\left(f_{j}^{(*)}\right)=0$ for all $1 \leq i \leq e$ and $1 \leq j \leq d$ by using the Leibniz rule. Furthermore, by using (4.5) and the fact $\nabla^{(0)}\left(f_{j}\right)=0$, we can deduce that we have $\nabla^{(0)}\left(f_{j}^{(*)}\right)=0$ for all $1 \leq j \leq d$.

(B) $\left\{f_{j}^{(*)}\right\}_{j=1}^{d} \in R[1 / t] \otimes_{R} D_{e-\text { dif }}^{+}(V)$ is linearly independent over $K_{\infty}^{(\mathrm{pf})}$. - By the presentation of $f_{j}^{(*)}$, we have

$$
f_{j}^{(*)}=f_{j}+g_{j} \quad\left(g_{j} \in\left(t_{1}, \ldots, t_{e}\right)\left(B_{\mathrm{dR}, K} \otimes_{\mathbb{Q}_{p}} V\right)\right) .
$$

Since $\left\{f_{j}\right\}_{j=1}^{d}$ forms a basis of $R[1 / t] \otimes_{R} D_{e \text {-dif }}^{+}(V)$ over $R[1 / t]$, it is, in particular, linearly independent over $K_{\infty}^{(\mathrm{pf})}(\subset R[1 / t])$. Thus, $\left\{\overline{f_{j}}={\overline{f_{j}}}^{(*)}\right\}_{j=1}^{d}$ (- denotes the reduction modulo $\left.\left(t_{1}, \ldots, t_{e}\right)\right)$ is linearly independent over $K_{\infty}^{(\mathrm{pf})}$ and we can see that $\left\{f_{j}^{(*)}\right\}_{j=1}^{d}$ is linearly independent over $K_{\infty}^{(\mathrm{pf})}$ in $R[1 / t] \otimes_{R} D_{e \text {-dif }}^{+}(V)$. 
(C) Conclusion. - Therefore, on the $K$-vector space generated by $\left\{f_{j}^{(*)}\right\}_{j=1}^{d}, \log (\gamma)$ and $\left\{\log \left(\beta_{i}\right)\right\}_{i=1}^{e}$ act trivially $\left(\Leftrightarrow \nabla^{(0)}\left(f_{j}^{(*)}\right)=0\right.$ and $\nabla^{(i)}\left(f_{j}^{(*)}\right)=0$ for all $1 \leq i \leq e$ and $1 \leq j \leq d)$. Thus, this means that $\Gamma_{K}$ acts on this $K$-vector space via finite quotient and there exists a finite field extension $L / K$ in $K_{\infty}^{(\mathrm{pf})}$ such that $\left\{f^{(*)}\right\}_{j=1}^{d}$ forms a basis of $D_{\mathrm{dR}, L}(V)$ over $L$. Since a potentially de Rham representation of $G_{K}$ is a de Rham representation of $G_{K}$, this completes the proof.

\title{
REFERENCES
}

[1] F. Andreatta, O. Brinon, $B_{\mathrm{dR}}$-representations dans le cas relatif, this volume, p. 279-339.

[2] O. Brinon, Une généralisation de la théorie de Sen, Math. Ann. 327 (2003), 793-813.

[3] O. Brinon, Représentations cristallines dans le cas d'un corps résiduel imparfait, Ann. Inst. Fourier (Grenoble) 56 (2006), 919-999.

[4] J.-M. Fontaine, Le corps des périodes p-adiques, Astérisque 223 (1994), 59-111.

[5] J.-M. Fontaine, Représentations p-adiques semi-stables, Astérisque 223 (1994), 113 184.

[6] J.-M. Fontaine, Arithmétique des représentations galoisiennes $p$-adiques, Astérisque 295 (2004), 1-115.

[7] O. Hyodo, On variation of Hodge-Tate structures, Math. Ann. 284 (1989), 7-22.

[8] K. Kato, Generalized explicit reciprocity laws, Adv. Stud. Contemp. Math. (Pusan) 1 (1999), 57-126.

[9] K. Kato, $p$-adic Hodge theory and values of zeta functions of modular forms, Astérisque 295 (2004), 117-290.

[10] S. Sen, Continuous cohomology and $p$-adic Galois representations, Invent. Math. 62 (1980/81), 89-116.

[11] T. Tsusi, Purity for Hodge-Tate representations, preprint.

[12] N. Tsuzuki, Variation of $p$-adic de Rham structures, preprint, 1991.

(Manuscrit reçu le 18 novembre 2008; accepté, après révision, le 28 septembre 2009.)

\author{
Kazuma Morita \\ Department of Mathematics \\ Hokkaido University \\ Sapporo 060-0810, Japan \\ E-mail: morita@math.sci.hokudai.ac.jp
}

